\title{
Direct helicopter EM - Sea-ice thickness inversion assessed with synthetic and field data
}

\author{
Andreas Pfaffling ${ }^{1}$, Christian Haas ${ }^{2}$, and James E. Reid ${ }^{3}$
}

\begin{abstract}
Accuracy and precision of helicopter electromagnetic (HEM) sounding are the essential parameters for HEM seaice thickness profiling. For sea-ice thickness research, the quality of HEM ice thickness estimates must be better than $10 \mathrm{~cm}$ to detect potential climatologic thickness changes. We introduce and assess a direct, 1D HEM data inversion algorithm for estimating sea-ice thickness. For synthetic quality assessment, an analytically determined HEM sea-ice thickness sensitivity is used to derive precision and accuracy. Precision is related directly to random, instrumental noise, although accuracy is defined by systematic bias arising from the data processing algorithm. For the in-phase component of the HEM response, sensitivity increases with frequency and coil spacing, but decreases with flying height. For small-scale HEM instruments used in sea-ice thickness surveys, instrumental noise must not exceed 5 ppm to reach ice thickness precision of $10 \mathrm{~cm}$ at $15-\mathrm{m}$ nominal flying height. Comparable precision is yielded at 30-m height for conventional exploration HEM systems with bigger coil spacings. Accuracy losses caused by approximations made for the direct inversion are negligible for brackish water and remain better than $10 \mathrm{~cm}$ for saline water. Synthetic precision and accuracy estimates are verified with drill-hole validated field data from East Antarctica, where HEM-derived level-ice thickness agrees with drilling results to within $4 \%$, or $2 \mathrm{~cm}$.
\end{abstract}

\section{INTRODUCTION}

As an indicator and a positively coupled variable of climate change, sea-ice extent and thickness distribution have been increas- ingly targeted in polar research over the last decade. The sea-ice thickness distribution and ice extent in the Arctic and Antarctic oceans are key parameters in understanding the effects of global warming (ACIA, 2004). Besides passive microwave remote sensing data for sea-ice extent (Stroeve et al., 2005), frequency domain electromagnetic induction has become widely used to study changes in the sea-ice thickness distribution (Haas, 2004).

Regional mapping of the sea-ice thickness distribution using helicopter electromagnetics (HEM) began in the late 1980s in North America, and was further developed in Canada prior to the latest research in Europe in the mid 1990s. Research on the applicability of helicopter EM for sea-ice studies was initiated in 1985 by the U. S. Army Corps of Engineers' Cold Regions Research and Engineering Laboratory (CRREL) (Kovacs et al., 1987). During those first attempts, using a conventional four-frequency mineral exploration HEM system, ice thickness profiles were retrieved with reasonable accuracy. The overall results were promising enough to develop a sea-ice-dedicated, small-scale, three-frequency sensor (Kovacs and Holladay 1990) and later a broadband system with frequencies up to $200 \mathrm{kHz}$ (Kovacs et al., 1995). Similar development took place at the Bedford Institute of Oceanography (BIO) in Canada cooperating with the Canadian Coast Guard. Field tests started with a conventional exploration system (Holladay et al., 1990) leading to a smallscale, two-frequency towed instrument (Peterson et al., 1999) and, most recently, a four-frequency helicopter-nose hard mounted system (Prinsenberg et al., 2002).

The first European airborne EM sea-ice field program was conducted in the Baltic Sea using the Geological Survey of Finland's (GSF) fixed wing EM system (Multala et al., 1996). After the reported campaigns in the winters of '91, '93, and '94, the GSF system has not been used for sea-ice thickness surveys. The latest European development was initiated in 2000 by the Alfred Wegener Institute for Polar and Marine Research (AWI) in Bremerhaven, Germany. The AWI HEM system is a small-scale, purpose-built, adaptable, fully

Manuscript received by the Editor April 12, 2006; revised manuscript received January 29, 2007; published online May 15, 2007.

${ }^{1}$ Formerly Alfred Wegener Institute for Polar and Marine Research, Climate Science Division, Sea Ice Physics Group, Bremerhaven, Germany; presently Consulting Geophysicist, Hamburg, Germany. E-mail: ap@ pfaffling-geophysics.com.

${ }^{2}$ Alfred Wegener Institute for Polar and Marine Research, Climate Science Division, Sea Ice Physics Group, Bremerhaven, Germany. E-mail: Christian.Haas@awi.de.

${ }^{3}$ Formerly University of Tasmania, School of Earth Sciences, Hobart, Tasmania, Australia; presently Geoforce Pty Ltd., Perth, Australia. E-mail: james@geoforce.com.au.

(C) 2007 Society of Exploration Geophysicists. All rights reserved. 
digital instrument that has been used on an operational basis on shipand land-based expeditions in the Arctic, Antarctic, and Baltic seas.

Distorted HEM data leads to difficulties with data processing, resulting in inconsistencies between geophysical and ground-truth data. Among further error sources, random instrumental noise degrades inversion results caused by the high sensitivity of the commonly used least-squares inversion to outlying data points (Meju, 1994). Kovacs et al. (1987) mention a decrease in level-ice-thickness error from $19 \%$ to $6 \%$ by improving the inversion parameters. Experience gained since 1990 at BIO leads to operational, real-time thickness inversion for their small-scale sea-ice profiler (Ice Probe, internal reports). As an alternative to elaborate integral inversion and to speed up and simplify inversion methods, Bergeron (1986) introduced a two-layer approximation of the analytic HEM response (modified image method, MIM). For a two-frequency HEM system, MIM converts in-phase and quadrature data directly to bird height, first-layer thickness and conductivity, as well as to the two-layer conductivity contrast. This method has proved successful for HEM bathymetry applications (Bergeron et al., 1989) and salinity mapping (Bryan et al., 2003). Besides an analytical feasibility study (Bergeron et al., 1987) there is no evidence for operational usage of MIM for sea-ice thickness mapping. For the sea-ice case, MIM appears to depend on an extremely high system frequency $(6.5 \mathrm{MHz})$ to achieve a skin depth smaller than the expected ice thickness.

Fundamentally simplifying sea-ice thickness HEM inversion, we present a direct HEM data to an ice thickness equation, termed the EMPEX transform (empirical exponential). The transform is based on a $1 \mathrm{D}$ approximation of the sea-ice thickness problem and a further exponential fit to layered-earth HEM response curves. A detailed discussion on the performance of direct inversion (EMPEX transform) in comparison to a suite of least-squares layered-earth inversion routines has been submitted to the Journal of Applied Geophysics by Pfaffling and Reid. Here we concentrate on the definition and assessment of precision and accuracy of the EMPEX-derived ice thickness estimates.

To provide a basic understanding of the theoretical precision and accuracy of sea-ice thickness measurements made using the AWI HEM system, we present an analytic sensitivity equation for the ice thickness case. Sensitivity is studied for the AWI geometry as well as conventional exploration HEM systems. The EMPEX transform is assessed with synthetic data for saline and brackish water conditions, simulating Arctic or Antarctic and Baltic or Caspian seas, respectively. A field data example from an expedition to East Antarctica in 2003 (Massom et al., 2006) is presented to underline the EMPEX performance compared to ground-truth data.

\section{INSTRUMENTATION — THE AWI HEM SYSTEM}

In contrast to commercially available multifrequency exploration HEM systems with bird lengths between 8 and $10 \mathrm{~m}$ and weights of up to $300 \mathrm{~kg}$, the AWI HEM system is a two-frequency instrument housed in a 3.4-m-long towed bird weighing slightly more than $100 \mathrm{~kg}$. The AWI system utilizes two horizontal coplanar transmitter-receiver loop pairs operating at $3.68 \mathrm{kHz}$ (f1) and $112 \mathrm{kHz}$ (f2) with coil separations of 2.77 and $2.05 \mathrm{~m}$, respectively. The bird is supplied with onboard calibration coils allowing phase and gain checks during every base level drift ascent. Ascents to $\sim 800 \mathrm{ft}$ are performed every 20 flight minutes to adjust the zero level of the measured secondary electromagnetic field (Valleau, 2000). Drift controls between these drift ascents are conducted frequently, when open water patches are crossed during ice thickness profiling. Comparing those measurements with their respective half-space model response guarantees sufficient drift linearity for post-flight correction. Data acquisition and preprocessing is conducted by a PC inside the bird, sampling the EM data at $10 \mathrm{~Hz}$ and the built-in laser altimeter at $100 \mathrm{~Hz}$. The 20-m-long towing cable solely connects to the helicopter's 28-V DC outlet, allowing the system to be carried by a broad variety of helicopter types. A wireless network connection between the bird PC and the operator laptop in the aircraft allows inflight bird control and data display. The system is usually flown at a nominal 15-m height at a speed of 60 to 80 knots. A detailed description of the system and its technical specialties and performance will be given elsewhere (paper submitted to the Journal of Applied Geophysics by Haas et al.). However, here we focus on the innovative processing method developed for the instrument.

\section{METHODS}

The basic principle of HEM sea-ice thickness profiling is to estimate the bird-to-water distance from the EM data, while a laser altimeter in the bird determines the system height above the ice or snow surface. The difference between these two distances consequently corresponds to the ice (or ice + snow) thickness. Whenever sea-ice thickness is mentioned in this paper, it actually refers to the total thickness - ice thickness plus snow thickness. There is no way to distinguish between snow and ice by HEM with the described system configuration.

When interpreting electromagnetic data for sea-ice thickness, generally two different ice types have to be considered. The first and more complicated case is deformed ice, so-called pressure ridges, where the ice floes have been broken up, crushed against each other, and finally piled up into distinct topographic features. Attempts to process HEM data related to these 3D features were made at the University of California, Berkeley by Liu and Becker (1990). These involved a compilation of interpretation charts for common pressure ridge shapes. Eventually, an elaborate $2 \mathrm{D}$ inversion scheme was presented (Liu et al., 1991). However, because of necessary extensive and advanced computing and only minor improvements in field results, multidimensional data processing is not yet used on an operational basis. Furthermore, the geometry of real-world pressure ridges is rarely as simple as presumed by the $2 \mathrm{D}$ models involved in the inversion. Being composed of a mixture of single, broken blocks of ice and ocean water, pressure ridges are very difficult to describe even by drill-hole thickness measurements. Therefore ground-truth data to validate processing algorithms cannot be acquired with the desired accuracy and detail. Idealized structure models of pressure ridges are sketched in Kovacs and Holladay (1990, Figure 5) showing the high keel porosity. The underestimation of pressure ridge thickness due to 1D EM processing is discussed by Reid et al. (2003).

In contrast to deformed ice, most sea ice is composed of homogeneous level ice, representing a computationally simpler 1D situation. Level-ice thickness depends on the thermodynamic growing conditions, but pressure ridges are linked to short-term events, e.g., storms. All approximations considered in this study focus on the determination of level-ice thickness.

\section{General HEM 1D forward modeling}

For a 1D subsurface geometry (layered half-space), the HEM response for the vertical dipole mode can be expressed as a Hankel 
transform utilizing a Bessel function of the first kind of order zero $\left(J_{0}\right)$, leading to

$$
Z=-r^{3} \int_{0}^{\infty} r_{T E} e^{-2 \lambda h} \lambda^{2} J_{0}(\lambda r) \mathrm{d} \lambda,
$$

where $r$ is the coil separation, $h$ the receiver and transmitter height above ground, and $\lambda$ the wavenumber. The recursively determined transverse electric (TE) mode reflection coefficient $r_{T E}$ is a function of system frequency and the electromagnetic properties of the conducting half-space (Ward and Hohmann, 1988). $Z$ is the normalized, secondary magnetic field at the receiver coil position usually expressed in parts per million. $Z=H_{z}^{S} / H_{z}^{P}$, where $H_{z}^{P}$ is the primary field (in a nonconductive full-space) and $H_{z}^{S}$ the secondary magnetic field (above a conductive half-space arising from the eddy currents induced by the primary field). For a homogeneous half-space, $r_{T E}$ can be expressed as a straightforward quotient and hence the layered half-space solution (equation 1) simplifies to

$$
Z=-r^{3} \int_{0}^{\infty} \frac{\lambda-\sqrt{\lambda^{2}+i \omega \sigma \mu}}{\lambda+\sqrt{\lambda^{2}+i \omega \sigma \mu}} e^{-2 \lambda h} \lambda^{2} J_{0}(\lambda r) \mathrm{d} \lambda .
$$

Here, the kernel includes the angular frequency of the EM field ( $\omega$ $=2 \pi f$ ), the electric conductivity $\sigma$, and magnetic permeability $\mu$ of the conducting half-space. The appearance of the imaginary unit $i$ in the homogeneous half-space solution (equation 2) underlines the complex nature of $Z$, usually described as in-phase (IP) and quadrature (Q) component or channel. In this study, the 1D models for layered and homogeneous half-space (equations 1 and 2) are computed by means of digital filtering (Guptasarma and Singh, 1997).

\section{Electric properties of sea-ice covered oceans}

Saline ocean water and brackish seawater represent distinct seaice environments, as they exhibit different electromagnetic target characteristics. The Arctic Ocean and the Southern Ocean surrounding Antarctica are characterized by saline ocean water with salinities around 35 PSU $( \pm 5)$, although brackish water prevails in the northern Baltic ( $<6$ PSU) and Caspian seas (10-13 PSU). The parameter of main interest is the conductivity of the water below the sea-ice cover, which is a function of salinity and water temperature. In polar conditions and in the presence of sea ice, the water temperature is commonly close to the freezing point, resulting in electric conductivities of 2.4-2.8 S/m for Arctic or Antarctic waters and $\sim 0.3$ or $\sim 1 \mathrm{~S} / \mathrm{m}$ for Baltic or Caspian waters.

The conductivity of sea ice is generally about two magnitudes smaller than the water from which it was formed because most of the brine is expelled from the ice while it freezes. Bulk ice conductivities between 20 and $50 \mathrm{mS} / \mathrm{m}$ can be presumed for newly formed firstyear (FY) ice (Timco, 1979). When an ice floe survives one summer melt season, almost all of its remaining enclosed brine has been drained out and the conductivity decreases by another order of magnitude. This makes it practically transparent for EM induction, in contrast to the highly conductive ocean. Based on sea-ice model studies, Morey et al. (1984) show that the bulk-ice conductivity may not exceed $50 \mathrm{mS} / \mathrm{m}$. Note that so far strictly bulk conductivity was addressed, which is not necessarily the parameter picked up by EM induction.

Because of both the growth structure of sea ice where brine cells assume a preferred vertical orientation and the subsequent development of vertical brine channels, level ice shows a strong vertical-to- horizontal conductivity anisotropy with the horizontal conductivity smaller than the vertical (Thyssen et al., 1974). The in situ conductivity of sea ice is hard to measure on samples because the conductive brine drains out of the ice structure when an ice core is drilled and taken to a lab. However, recent in situ DC-resistivity measurements in Antarctica (Reid et al., 2006b) indicate an even smaller horizontal conductivity than usually expected, averaging $17 \mathrm{mS} / \mathrm{m}$.

For the synthetic HEM data presented in this paper, seawater and sea-ice conductivities of 2.767 and $50 \mathrm{mS} / \mathrm{m}$, or 0.3 and $1 \mathrm{mS} / \mathrm{m}$, were used for Antarctic and Arctic (Ant-/Arctic) or Baltic conditions, respectively. For comparison with earlier studies, we decided to use $50 \mathrm{mS} / \mathrm{m}$ as polar sea-ice conductivity, rather than the actual lower values from in situ measurements.

\section{Approximations involved}

For HEM sea-ice thickness mapping, the bird altitude over the conductive ocean water is the model parameter of interest. To develop a direct-inversion method to derive the distance to water, three main approximations are made.

\section{Conductive seawater half-space}

The seawater conductivity is assumed to be known and constant. Though the water salinity and consequently conductivity may change significantly on a regional scale, it can be assumed as constant within a certain survey area. As an example, the measured seawater conductivity statistics along the track north of $80^{\circ}$ latitude of an RV Polarstern expedition to the northern Fram Strait (Schauer and Kattner, 2004) result in an average of $2.713 \mathrm{~S} / \mathrm{m}$, with $0.042 \mathrm{~S} / \mathrm{m}$ standard deviation. Thermosalinometer data of RV Aurora Australis (Massom et al., 2006) acquired in the east Antarctic result in an average seawater conductivity of $2.769 \mathrm{~S} / \mathrm{m}$, with $0.054 \mathrm{~S} / \mathrm{m}$ standard deviation. Significantly different seawater conductivities may exist in distinct oceanographic regions (e.g., Lincoln Sea, 2.4 S/m, unpublished AWI field campaign). However, when the local water conductivity is determined, it is likely to be constant if there are no disturbing features, such as river mouths or other oceanographic anomalies.

\section{Resistive sea-ice layer}

The sea-ice conductivity is neglected, making the ice transparent to HEM induction. Because of the pronounced contrast between the seawater and ice conductivity of two to three orders of magnitude and the small ice thickness compared to the bird height, the majority of the induced eddy currents flow in the conductive seawater. Model studies comparing synthetic data for conductive and transparent ice are shown in the accuracy section, proving that, for ice of moderate thickness $(2-3 \mathrm{~m})$, the effect of its conductivity is negligible for low frequencies.

\section{Model dimensionality}

The sea-ice structure is simplified to a $1 \mathrm{D}$ problem. As discussed before, the thermodynamic history of the sea-ice cover and the levelice thickness is the key parameter. Obviously, 2D and 3D features in the ice structure will be smoothed because of the $1 \mathrm{D}$ processing and the footprint size of the induction process (Reid et al., 2003). Lateral smoothing has a minor effect on ice thickness distribution function, which is used to determine regional level-ice thickness and is introduced in the EMPEX assessment section. 


\section{Limitations}

The vast majority of common situations in sea-ice thickness mapping allows for the use of EMPEX approximations. However, rare or extreme sea-ice conditions may exist, where the assumptions are not valid, and thus, EMPEX may yield biased sea-ice thickness estimates. One extreme condition met in late Antarctic summer is the development of gap layers - highly porous partially melted layers near the ice surface. Gap layers are highly conductive (close to sea water conductivity) and, therefore, bias direct inversion results toward underestimated thickness. A rarely met problem in polar oceans is shallow water, which would rule out the approximation of the conductive seawater by a half-space. In the shallow northern Baltic Sea, biased ice thickness is evident for water depths less than $10 \mathrm{~m}$. Direct inversion cannot account for bird attitude (roll-andpitch) variations, which may have significant influence on the thickness estimates, mainly caused by the tilted laser altimeter (Holladay

a)

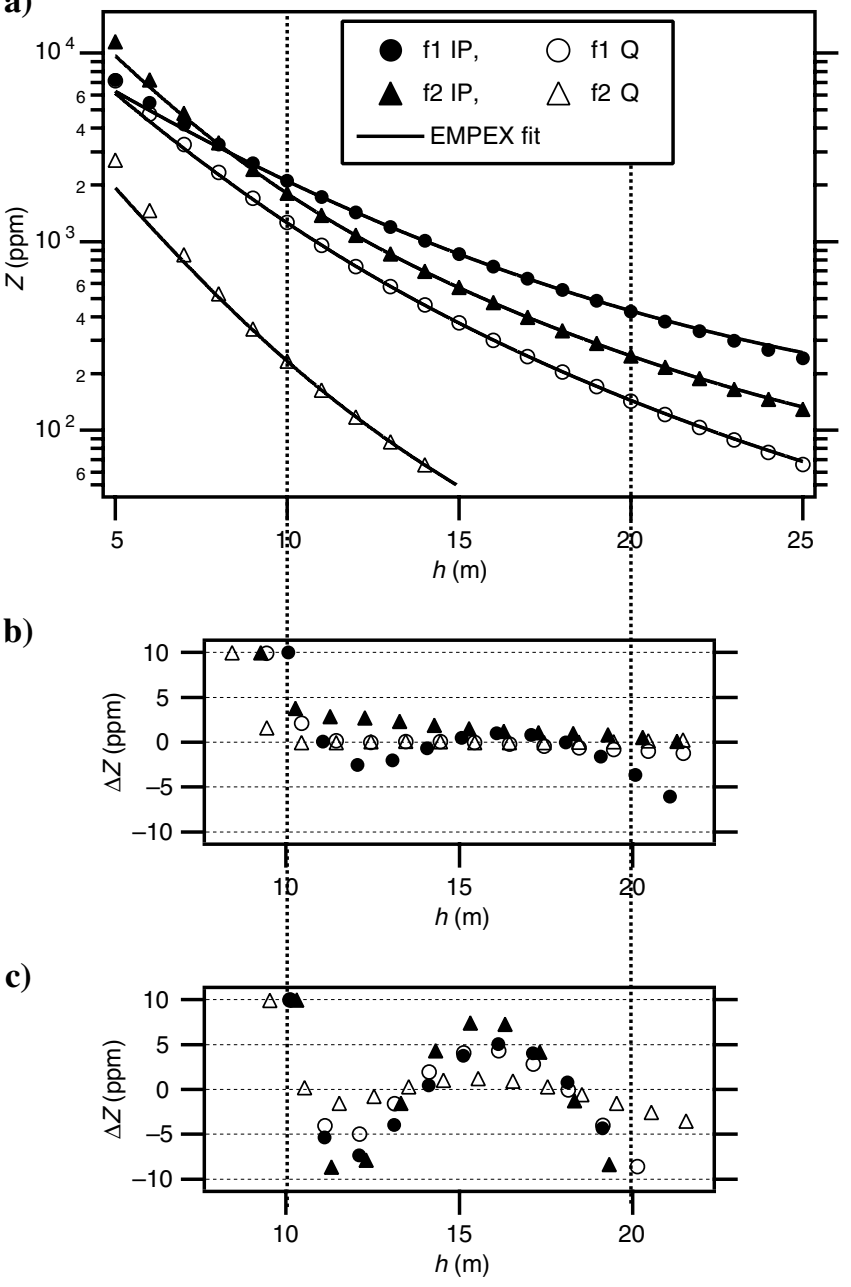

Figure 1. (a) HEM response over a conductive half-space (markers), modeled for the AWI bird frequencies $3.68 \mathrm{kHz}$ (f1) and $112 \mathrm{kHz}$ (f2) with respect to bird height (h) and further fitted with the secondorder EMPEX approximation (lines). Panels (b) and (c) show the residual between EMPEX fit and forward model for the second- and first-order approximation, respectively. The marker legend in (a) also applies for (b) and (c). Because the EMPEX fit is computed for $10<h<20 \mathrm{~m}$, (b) and (c) are shortened accordingly. et al. 1997). Layered-earth inversion would be able to account for sea-ice conductivity variations as well as shallow bathymetry, given a suitable set of frequencies and coil spacing. If bird attitude is measured, it can be included in the inversion procedure.

\section{Toward a direct inversion}

Considering the introduced approximations in the half-space solution, equation 2 simplifies to a function solely dependent on the bird height $h$. Hence applying numerical integration, e.g., NewtonCotes formulas (Abramowitz and Stegun, 1964, Section 25.4), the Hankel transform can be approximated by a series of exponential functions:

$$
Z(h) \approx \sum_{i=0}^{n} B_{i} e^{C_{i} h}
$$

Reducing the series to $n=2$ with $C_{0}=0$ leads to

$$
Z(h) \approx B_{0}+B_{1} e^{-C_{1} h}+B_{2} e^{-C_{2} h} .
$$

Equation 4 is here called the second-order approximation (as $n=2$ ) to the layered half-space response (equation 2). The coefficients $B_{0}$, $B_{1}, B_{2}, C_{1}$, and $C_{2}$ are determined by exponential fitting to synthetic half-space model curves within a given height range. Figure 1 illustrates the fitting quality and gives a comparison to the first-order EMPEX approximation $\left(Z \approx B_{0}+B_{1} e^{-C_{1} h}\right)$, which is commonly used for EM31 ice thickness estimation (Haas et al., 1997). The exponential fit in Figure 1 is run for a 10-20-m flying height range.

Although the inverse of the first-order approximation could be determined as an explicit logarithmic equation, the required model parameter $h$ (the distance from bird to sea water surface) in the secondorder approximation (equation 4) is evaluated with a root-finding algorithm using a Lagrange interpolation polynomial (Brent, 1973). Hence the distance between bird and water surface $h_{E M}$ can be determined for any measured EM field $Z$ in an unambiguous, numerically robust way. The ice thickness is consequently derived by

$$
z_{i}=h_{E M}-h_{L}
$$

where $h_{L}$ is the bird height measured by the laser altimeter and $z_{i}$ the ice (total) thickness.

\section{Sensitivity}

In geophysical inversion, the Jacobian matrix describes the system's sensitivity to specific model parameters, consisting of partial derivatives of measured data with respect to all model parameters. For a layered half-space HEM response (equation 1), the sensitivity $S$ with respect to $h$, the parameter of interest in this case, can be expressed analytically. The partial derivative of $Z$ with respect to $h$ for a homogeneous half-space leads to equation 6, describing the HEM sensitivity to the bird height over ocean water:

$$
S=\frac{\delta Z}{\delta h}=2 r^{3} \int_{0}^{\infty} \frac{\lambda-\sqrt{\lambda^{2}+i \omega \sigma \mu}}{\lambda+\sqrt{\lambda^{2}+i \omega \sigma \mu}} e^{-2 \lambda h} \lambda^{3} J_{0}(\lambda r) \mathrm{d} \lambda .
$$




\section{RESULTS}

The sensitivity (equation 6) provides the opportunity to study, in theory, the utility of HEM for sea-ice thickness mapping. Two profoundly different data quality measures are described in this section, precision and accuracy. The former is governed by instrumental system noise, translated to precision estimates using determined sensitivity values. In contrast to the precision, residuals introduced by the EMPEX approximations relate to the method's accuracy. Finally, the EMPEX transform is used on synthetic data sets to assess the quality of the determined sea-ice thickness estimates. This leads to the final assessment of the method, which determines the superposition of the effects of limited precision resulting from system noise and decreasing accuracy caused by biases introduced by the approximations.

\section{Precision resulting from instrumental noise}

To investigate the theoretically achievable precision in sea-ice thickness estimates, sensitivity studies have been conducted for the major sea-ice environments. Highly saline Arctic and Southern ocean water as well as brackish Baltic water were considered. Sensitivity was computed analytically (equation 6) at typical flying heights for small-scale sea-ice birds, such as the AWI HEM system as well as conventional exploration birds.

\section{Sensitivity and precision for a small bird}

In climate research, the targeted ice thickness accuracy is $10 \mathrm{~cm}$. Presuming an instrumental noise level of 5 ppm leads to a sensitivity threshold of $50 \mathrm{ppm} / \mathrm{m}$ to meet the required precision. The dashed line in Figure 2 shows the $50 \mathrm{ppm} / \mathrm{m}$ threshold and consequently illustrates the maximum bird heights for the distinct channels. It further points out the necessity of small instrumental noise levels to keep the maximum flying height in a safe range for field operations.

Governed by water conductivity, the maximum sensitivity channel is the 3.68-kHz IP for Ant-/Arctic and 112-kHz IP for Baltic waters (Figure 2). These sensitivities are with respect to height above a homogeneous half-space. To account for finite sea-ice conductivity, a set of two-layer cases was investigated to determine sensitivity with respect to first-layer thickness. Table 1 provides results for an 18-m bird above water surface height. With a skin depth significantly larger than the ice thickness, $\mathrm{f} 1$ is not affected by the conductivity of the thin ice layer. The two-layer model results coincide with the half-space curve. In contrast to the validity of the half-space approximation for f1 IP and Q and f2 IP, the conductive ice layer influences the high-frequency quadrature. However, the $112-\mathrm{kHz}$ Q sensitivity is well below the noise level, even for the half-space model, and therefore not recommended for ice thickness retrieval.

\section{Universal sensitivities}

For a more detailed understanding and a better comparison to common HEM geometries, sensitivities, as introduced before, were determined for a broad frequency and coil-spacing range. Compared with conventional exploration systems, the technical and geophysical challenge in sea-ice thickness retrieval is the small bird size and low flying altitude. Analyzing the system sensitivities reveals some fundamental characteristics. Sensitivity was determined within a range of $100 \mathrm{~Hz}$ to $1 \mathrm{MHz}$ system frequency and $0.5-10-\mathrm{m}$ coil spacing for Ant-/Arctic (Figure 3a) as well as Baltic (Figure 3b) con- ditions at sensor heights of 15 and $30 \mathrm{~m}$. The highest and lowest frequency (with respective coil spacing) for the following four HEM systems are spotlighted in the graphs: (1) the AWI minibird; (2) a small-scale sea-ice bird, BIO'S Ice Probe (Peterson et al., 1999); (3) the conventional exploration bird used by CRREL in 1985 (Kovacs et al., 1987); and (4) Fugro Airborne Survey's RESOLVE bird, a modern six frequency exploration system (Smith et al., 2003).

Extending the findings for the AWI geometry, the sensitivity is a function of frequency and coil spacing $r$. The higher $S$ for $\mathrm{f} 1 \mathrm{IP}$ com-

a)

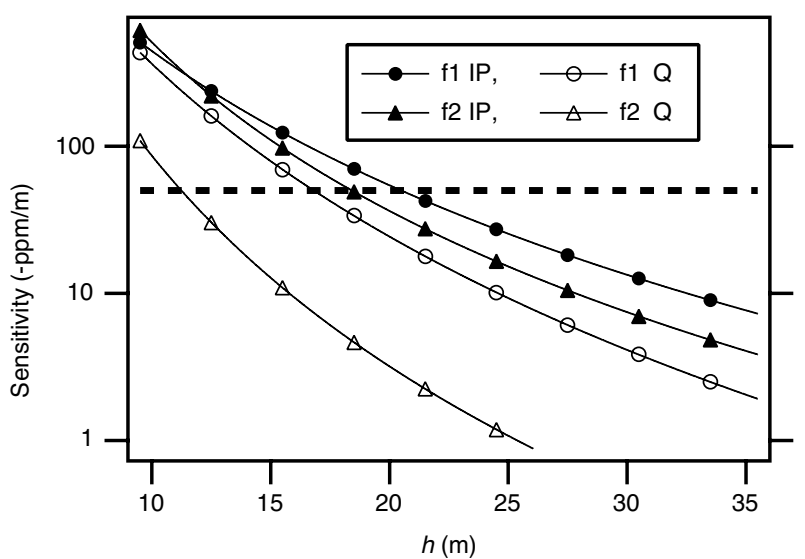

b)

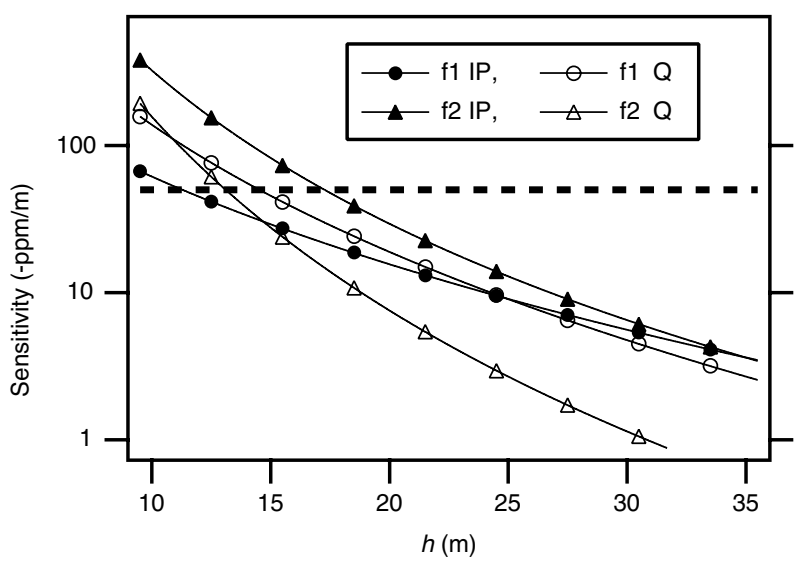

Figure 2. AWI bird sensitivities for (a) Ant-/Arctic and (b) Baltic conditions versus bird height $(h)$ for a homogeneous half-space model. The dashed line is at a potential noise level threshold of $-50 \mathrm{ppm} / \mathrm{m}$ (10 $\mathrm{cm}$ precision for $5 \mathrm{ppm}$ ambient noise $)$.

Table 1. Collection of AWI bird sensitivities with respect to ice thickness for a set of two-layer cases with constant cumulative distance between bird and water surface.

Sensitivity with respect to ice thickness $\left(z_{i}\right)(\mathrm{ppm} / \mathrm{m})$

\begin{tabular}{cccccr}
\cline { 3 - 5 } $\begin{array}{c}h \\
(\mathrm{~m})\end{array}$ & $\begin{array}{c}z_{\mathrm{i}} \\
(\mathrm{m})\end{array}$ & $3.68-\mathrm{kHz}$ IP & $3.68-\mathrm{kHz} \mathrm{Q}$ & $112-\mathrm{kHz}$ IP & $112-\mathrm{kHz} \mathrm{Q}$ \\
\hline 18 & 0 & 75.10 & 36.99 & 53.49 & 5.21 \\
17 & 1 & 75.05 & 36.29 & 51.95 & 1.09 \\
16 & 2 & 75.09 & 35.51 & 49.92 & -5.87 \\
15 & 3 & 75.18 & 34.65 & 46.06 & -14.42
\end{tabular}


pared to f2 IP in Figure 2a is the result of higher coil spacing rather than lower frequency. The coil spacing dominates the IP sensitivity for frequencies above $10 \mathrm{kHz}$. While the IP sensitivity generally increases steadily with $f$ and $r$, the $Q$ sensitivity peaks at a discrete frequency for given $r$. For f1, the very large coil spacing of the RESOLVE bird balances the higher operating altitude, resulting in $S$ comparable to the AWI bird at 15-m height for the lowest frequency

a)

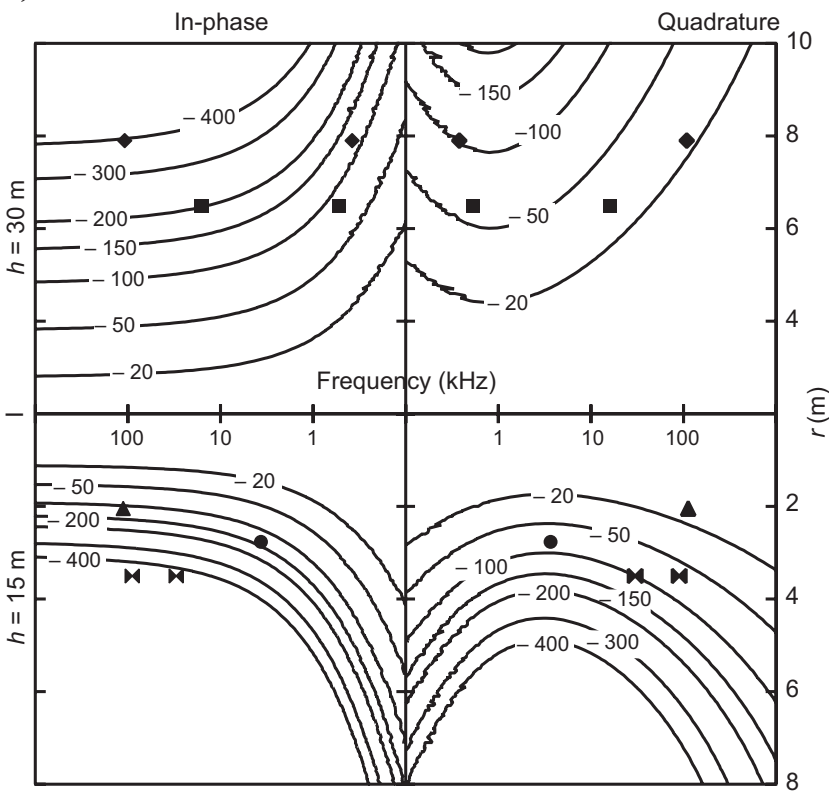

b)

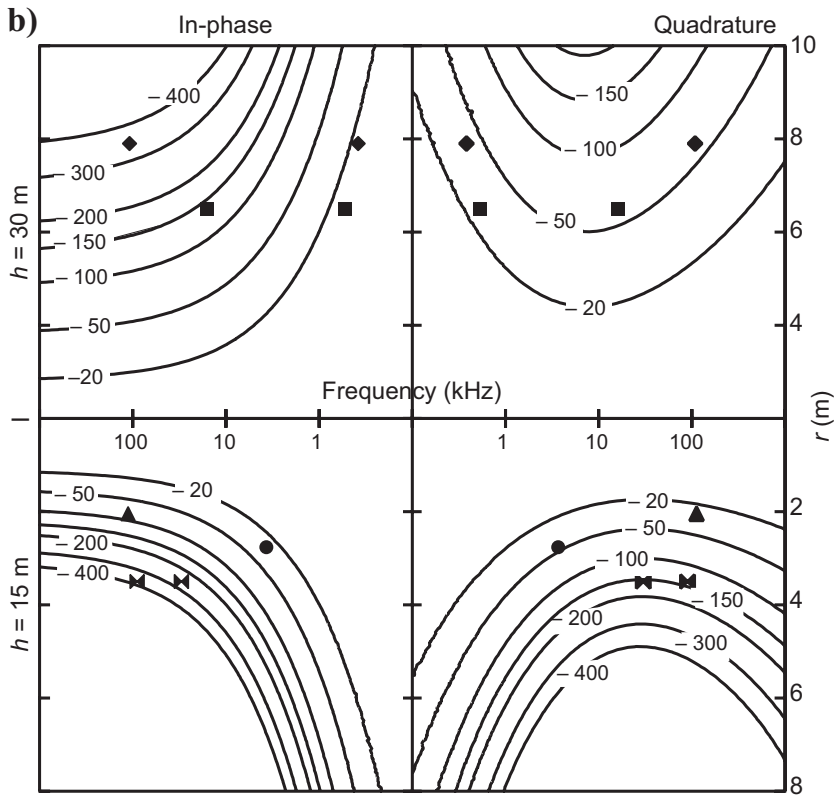

Figure 3. Maps of sensitivity with respect to bird height $(\delta Z / \delta h)$ for frequencies from $100 \mathrm{~Hz}$ to $1 \mathrm{MHz}$ and coil spacing $r$ from 0.5 to $10 \mathrm{~m}$. IP sensitivities are found in the left column; $\mathrm{Q}$ on the right. The upper row of the panels shows results for a bird height of $30 \mathrm{~m} ; \mathrm{h}=15 \mathrm{~m}$ is given in the lower row. Half-space conductivity is $2.767 \mathrm{~S} / \mathrm{m}$ for Ant-/Arctic and $0.3 \mathrm{~S} / \mathrm{m}$ for Baltic waters, presented in (a) and (b), respectively. Markers indicate $f-r$ parameters of four HEM systems' highest and lowest frequency: the AWI system ( $\mathrm{f} 1$, $\Delta \mathrm{f} 2$ ); the BIO sea ice thickness bird ( $)$; the early CRREL system $(\boldsymbol{\square})$; and the recent RESOLVE bird $(\bullet)$.
(AWI $\sim 140 \mathrm{ppm} / \mathrm{m} ;$ RESOLVE $\sim 120 \mathrm{ppm} / \mathrm{m}$ ). The low frequency of the CRREL bird results in a sensitivity of $\sim 80 \mathrm{ppm} / \mathrm{m}$, which makes it less suitable for EMPEX processing. However, the lowest of the four CRREL frequencies was designed for successful subice bathymetry mapping, which can be achieved using layered-earth inversion. The comparably large footprint at $h=30 \mathrm{~m}$ (Reid et al., 2006a) may explain the unsatisfactory sea-ice thickness results obtained with conventionally sized birds (Kovacs et al., 1987). Sensitivities for the vertical coplanar and vertical coaxial channels in the CRREL and RESOLVE birds were computed for comparison and were generally smaller than the horizontal coplanar channels with comparable coil spacing (not shown).

\section{The accuracy of an approximation}

Applying the determined sensitivities to the bias caused by treating sea ice as electromagnetic transparent, the second approximation made for the EMPEX transform can be quantified. Neglecting the sea-ice conductivity introduces a residual $(R[\mathrm{ppm}])$, which is used to express an EMPEX transform accuracy $A$ estimated by the quotient of $R$ and $S(A=R / S[\mathrm{~m}]) . R$ is defined as the difference between the half-space and the two-layer solution determined using two models with equal distance to water as in Table 1. As an example, $R$ for the Arctic is determined by $R=Z_{h}-Z_{i}$, where $Z_{h}$ is the half-space response at $h=15 \mathrm{~m}$ and $Z_{i}$ the result for a two-layer case with $h=12 \mathrm{~m}$ above a 3 -m-thick, conductive $(0.05 \mathrm{~S} / \mathrm{m})$ ice layer — both on a 2.767-S/m ocean. For accuracies shown in Figure 4, an ice thickness of 3 and $2 \mathrm{~m}$ was used for Ant-/Arctic and Baltic environments, respectively.

Because both $R$ and $S$ are mainly functions of $r^{3}$ (besides the $r$ in the Bessel function), the accuracy mainly depends on the frequency (equations 1 and 6). For the range of $A$ in Figure 4, the difference in accuracy for 2- or 8-m coil spacing is less than 3\%; hence, only 2-m results are shown here. The decrease of accuracy (increase in number means decrease in quality) with increasing frequency is mainly driven by the strongly rising residual toward higher frequency, although the sensitivity's slope levels out for IP or even declines in the case of Q. The accuracy generally suffers from high bird altitude and cannot be improved by increased coil spacing such as the case for the sensitivity. To meet the respective 15-m accuracy at higher altitude, the frequency has to be decreased, leading to lower sensitivity and consequently worse precision, in addition to the larger footprint at high altitude.

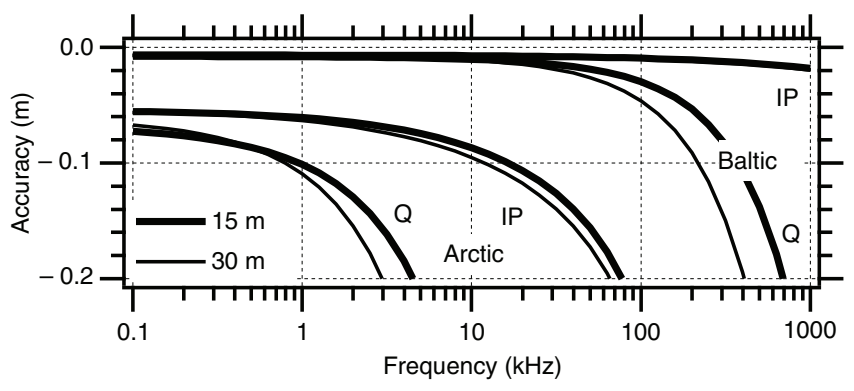

Figure 4. EMPEX accuracy versus system frequency, arising from the residual introduced by the half-space approximation. Accuracy was derived from residual and sensitivity $(A=R / S)$. IP and Q accuracies are presented at bird heights of 15 and $30 \mathrm{~m}$ for Ant-/Arctic and Baltic conditions. 
Precision and accuracy reveal opposite correlations with system frequency as far as the in-phase component is concerned. For the quadrature, however, an optimal frequency with maximum precision exists, although the algorithm accuracy decreases with frequency as it is for IP. Consequently, finding the right geometry-frequency trade-off is the main problem in sea-ice geophysics. The quadrature precision seems useful for choosing an optimal system frequency. A strong Q sensitivity would provide EMPEX results with high lateral resolution because of the smaller footprint of Q compared to IP (Reid et al., 2006a). The system geometry is usually limited by operational aspects. A low-flying system with a large transmitter-receiver separation would be favorable, but rather challenging for engineers and pilots and for operations on icebreakers. However, increasing the spacing of the AWI bird's $3.68 \mathrm{-kHz}$ coils from 2.77 to $3.5 \mathrm{~m}$ (like BIO's IceProbe) would approximately double the IP sensitivity.

\section{Synthetic assessment of the EMPEX transform}

To study the discussed effects of accuracy and precision on the EMPEX method, synthetic data were EMPEX transformed and are analyzed as follows. The EM response was modeled for a sinusoidally varying flying height between 10 and $20 \mathrm{~m}$ over a 3-m-thick ice layer floating on saline ocean water. Three different cases were studied. In case I, the ice conductivity was set to zero, simulating the half-space approximation; in case II, conductive ice was included with $50 \mathrm{mS} / \mathrm{m}$; and in case III, Gaussian noise was added to the fields obtained for case II with standard deviation $6.4,5.8,9.2$, and $10 \mathrm{ppm}$ for f1 IP, f1 Q, f2 IP, and f2 Q, respectively, representing typical field conditions (taken from the flight introduced in the field-data section). For clarity, the model ice thickness was subtracted from
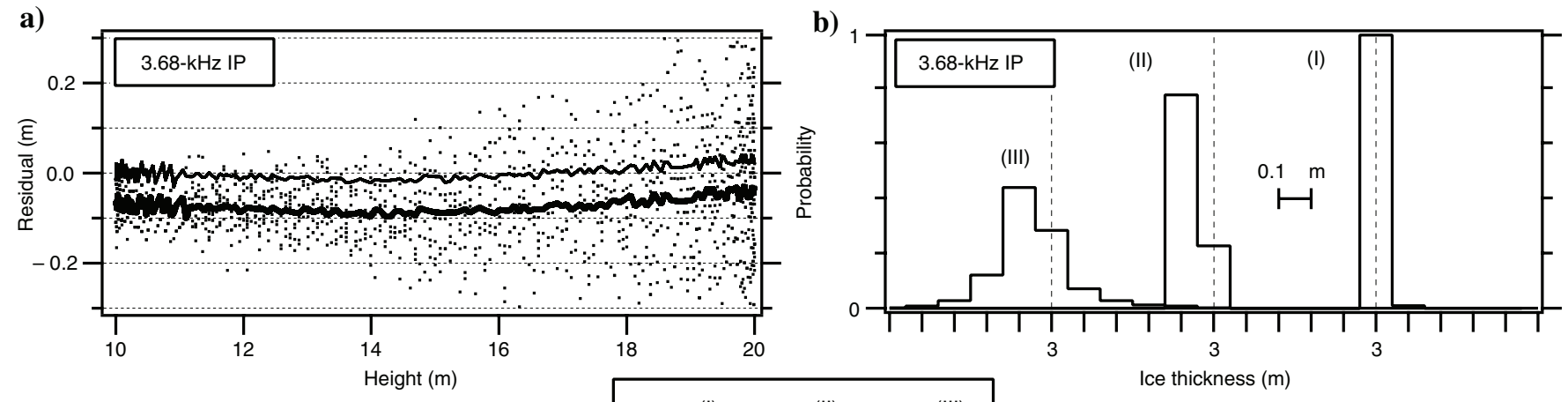

c)
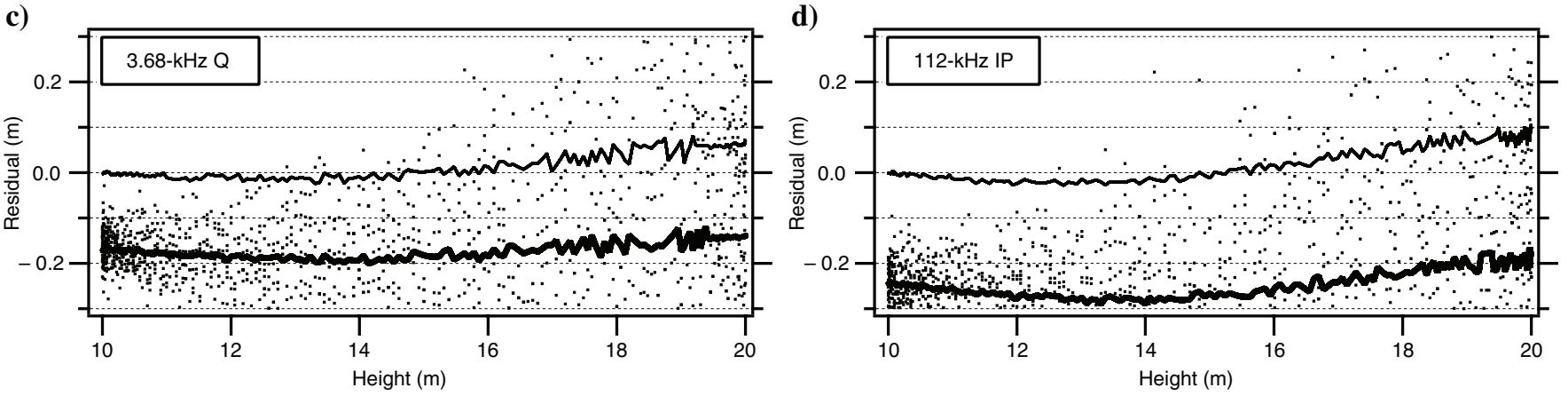

Figure 5. EMPEX ice thickness results from synthetic data with 3-m model ice thickness for varying bird altitudes. Panels (a), (c), and (d) show the residual between EMPEX thickness results and the model thickness. Each panel includes three different model runs: (I) electromagnetic transparent ice $(0 \mathrm{~S} / \mathrm{m})$, (II) conductive ice $(50 \mathrm{mS} / \mathrm{m})$, and (III) conductive ice as in (II) with Gaussian noise added. Panel (b) shows the ice thickness distribution obtained from f1 IP thickness in panel (a). The histogram bin size is $10 \mathrm{~cm}$. 
histograms peak at the correct thickness within a tolerance of $10 \mathrm{~cm}$. The high noise added in case III broadens the distribution, but does not bias the mode.

It needs to be stressed that the presented modeled accuracy examples represent worst-case conditions. To encounter ice with horizontal conductivities of $50 \mathrm{mS} / \mathrm{m}$ is highly unlikely and, it is even more unlikely to encounter 3-m-thick ice with high conductivities.

\section{Ant-/Arctic}

The EMPEX performance for increasing ice thickness was assessed with synthetic data computed according to the model drafted in Figure 6. A total of 6000 measurements for ice thickness from 0 to

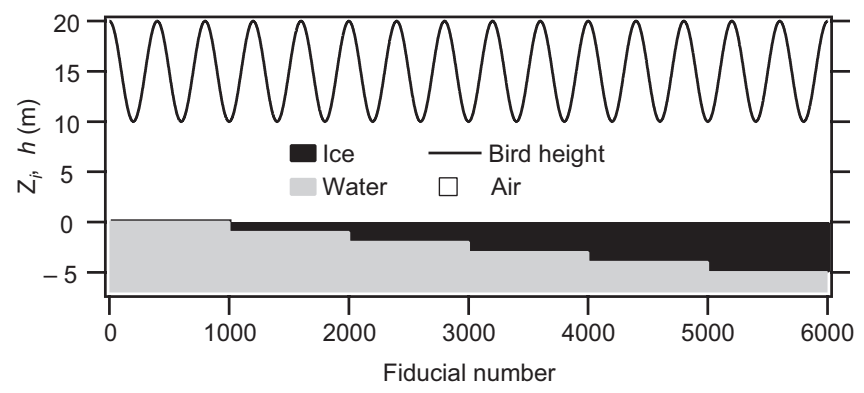

Figure 6. Model parameters used for synthetic EMPEX assessment studies. System height varies sinusoidal between 10 and $20 \mathrm{~m}$. Simulated ice thickness includes $0 \mathrm{~m}$ imitating open water, rising up to 5-m-thick level ice. Though the sketch implies a 2D structure, strictly $1 \mathrm{D}$ forward modeling has been engaged in this study.

\section{a)}

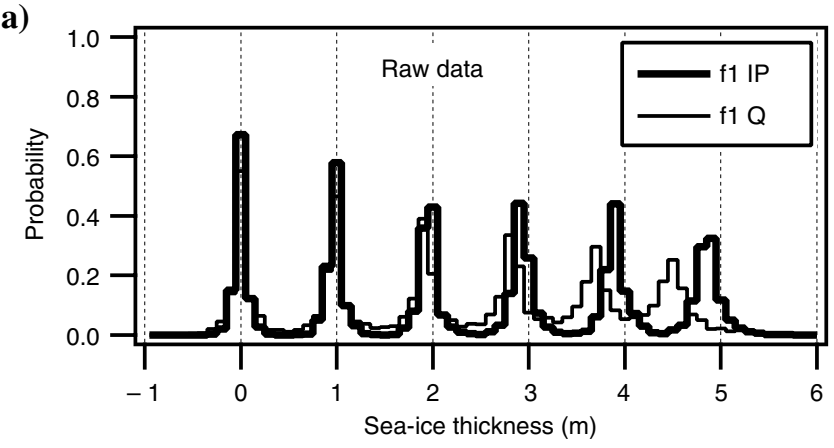

b)

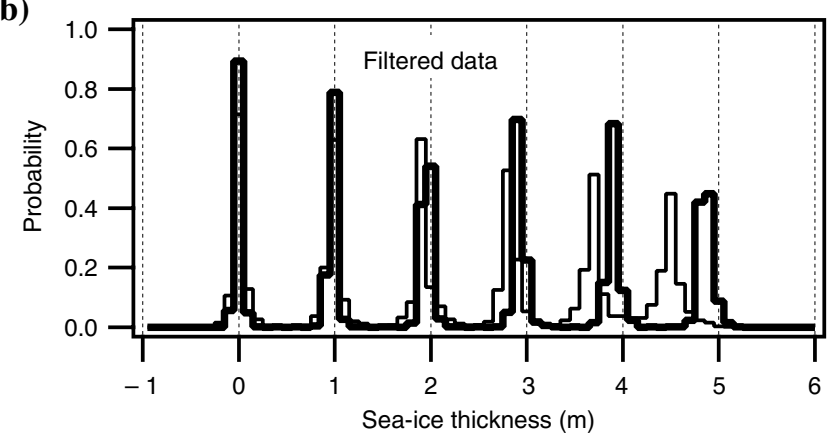

Figure 7. Ice thickness distribution of EMPEX-transformed synthetic data with added noise using model parameters shown in Figure 6 modeled for Ant-/Arctic conductivities. In both (a) and (b) $3.68-\mathrm{kHz}$ IP and Q are shown. The noisy data leading to the EMPEX thickness distribution in (a) have been filtered with a 5-point running average prior to transformation resulting in (b).
$6 \mathrm{~m}$ with varying bird height were modeled incorporating Ant-/Arctic conductivity parameters with added Gaussian noise as in case III in Figure 5. The histograms calculated for the low-frequency IP and $\mathrm{Q}$ (Figure 7) point out the higher accuracy of IP as suggested before (bias between distribution mode and model thickness), although the precision of IP and Q is comparable (width of the distribution peaks). Filtering the raw EM data with a five point running average prior to EMPEX transformation (Figure 7b) has no effect on the position of the mode (accuracy). However, the signal-to-noise ratio and precision in the histogram improves.

\section{Baltic}

Synthetic data for Baltic conditions with ice thickness from 0 to $2 \mathrm{~m}$ analyzed as they were for the Ant-/Arctic case result in comparable ice thickness distributions (not shown). Because the sensitivity is smaller for the brackish water, the histograms are broader and the bias resulting from ice conductivity is sufficiently small (within $10 \mathrm{~cm}$ for 2-m-thick ice). From the precision and accuracy sections (Figures 2 and 4), it is determined that, for brackish water, the highfrequency IP provides the best signal-to-noise ratio in the histogram - approximately two times the peak of f1 IP.

\section{FIELD DATA}

In September and October 2003, an Australian-led international experiment, dedicated to sea-ice remote sensing validation (Massom et al., 2006), took place onboard the icebreaker RV Aurora Australis in the east Antarctic marginal sea-ice zone. During a three-day experiment, almost 1000 drill-hole ice thickness measurements were made on three parallel 500-m-long, 20-m-spaced profiles, offering a unique data set for obtaining ground truth for airborne EM data. For optimum validation data, a level-ice floe with a prominent pressure ridge was chosen for this experiment. Ice-core analyses and DC soundings (Reid et al., 2006b) showed that the internal sea-ice structure was homogeneous, lacking any disturbing features, e.g., highly conductive surface or slush layers. HEM data were acquired along the central drill-hole profile at an average bird altitude of $14.7 \mathrm{~m}$ (15.1 $\mathrm{m}$ over water surface) and an operational speed of 60 knots $(30 \mathrm{~m} / \mathrm{s})$. To colocate HEM and drill-hole data, GPS readings were taken on the drifting ice floe at the moment the bird passed over the beginning and end of the line.

EMPEX thickness estimates from raw and filtered (five-point running average as in Figure 7) HEM data agree with drilled thickness data, particularly along the $\sim 0.5$-m-thick level-ice areas (Figure 8). Being fairly thin and moderately saline, the level ice introduces no residual affecting the accuracy of the EMPEX transform. The underestimated level-ice thickness between 90 and $130 \mathrm{~m}$ appears to be a bird-swing effect. The mean errors from measurements solely over level ice are $-0.04 \mathrm{~m} \pm 0.09 \mathrm{~m}$ and $-0.07 \mathrm{~m} \pm 0.09 \mathrm{~m}$ for $\mathrm{f}_{1}$ IP and $\mathrm{f}_{1} \mathrm{Q}$, respectively.

As anticipated, the massive 3D pressure ridge thickness is underestimated by $50 \%$ using the $1 \mathrm{D}$ processing method. Note that the steeper slope of the quadrature thickness in the vicinity of the major pressure ridge at 300-400 $\mathrm{m}$ is the result of the smaller footprint of Q (36 m) compared with IP (69 m) (Reid et al., 2006a). However, though being smaller than the footprint, the narrow ridge at $80 \mathrm{~m}$ is observable in the derived ice thickness. This is solely the result of the ridge topography, profiled by the laser, rather than the EM induction process. 
Finally, comparing ice thickness histograms from drilling data as well as IP and Q, EMPEX estimates prove the accuracy and precision of the EMPEX method (Figure 9). Even at 2-cm bin size, EMPEX and drilling histograms yield the same modal thickness. Filtering the raw EM data has a smaller effect on field data than on synthetic data, as instrumental and glaciological noise (e.g., the surface roughness picked up by the laser altimeter) interfere. The precision of the histogram (width of the distribution) does not improve with filtering. The clear level-ice thickness peak in the drilling histogram is wider in the HEM results. This arises from the predicted estimates (Figures 5 and 7), as well as bird-swing effects (especially at profile 90-130 m).

\section{DISCUSSION}

Applying the EMPEX transform to the full HEM data set results in a set of ice-thickness estimates. Generally these thicknesses should coincide, as it does along the level ice in Figure 8 for

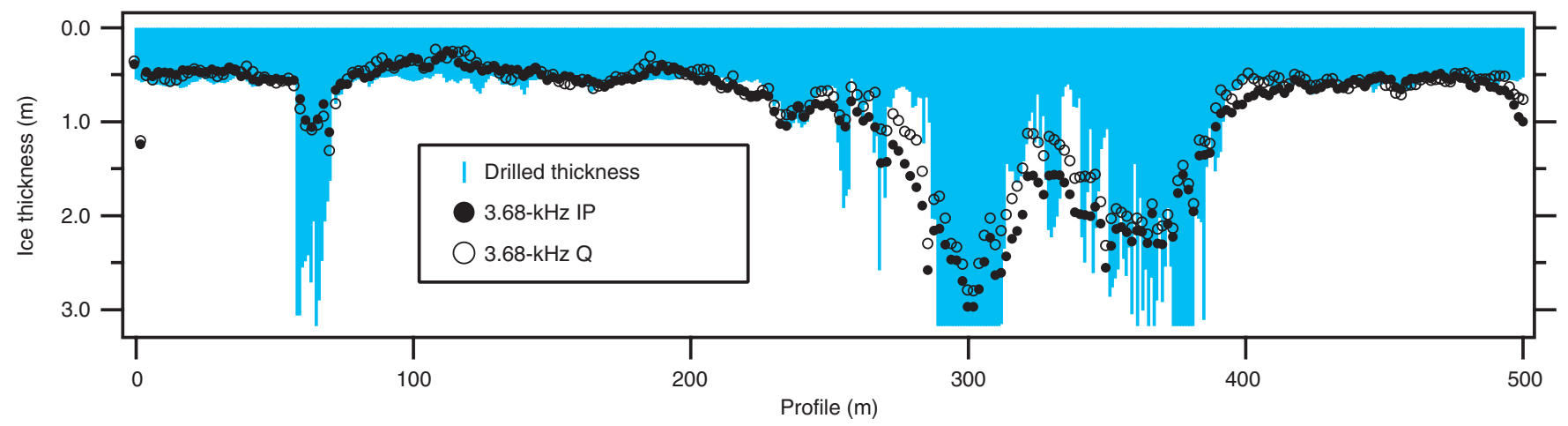

Figure 8. ARISE 2003 field data: comparison of ice thickness estimates from auger measurements and EMPEX-transformed HEM data. Drill spacing varied between 2 and $1 \mathrm{~m}$ along level ice or pressure ridge sections, respectively. The sampling frequency of the AWI bird is $10 \mathrm{~Hz}$, corresponding to $\sim 3 \mathrm{~m}$ point spacing. Two parallel drill-hole profiles, $20 \mathrm{~m}$ apart to both sides of the plotted line, and aerial photography imply strong lateral inhomogeneities in the main ridge structure. The graph does not display the maximum 5.8-m drilled ridge thickness at $305 \mathrm{~m}$.

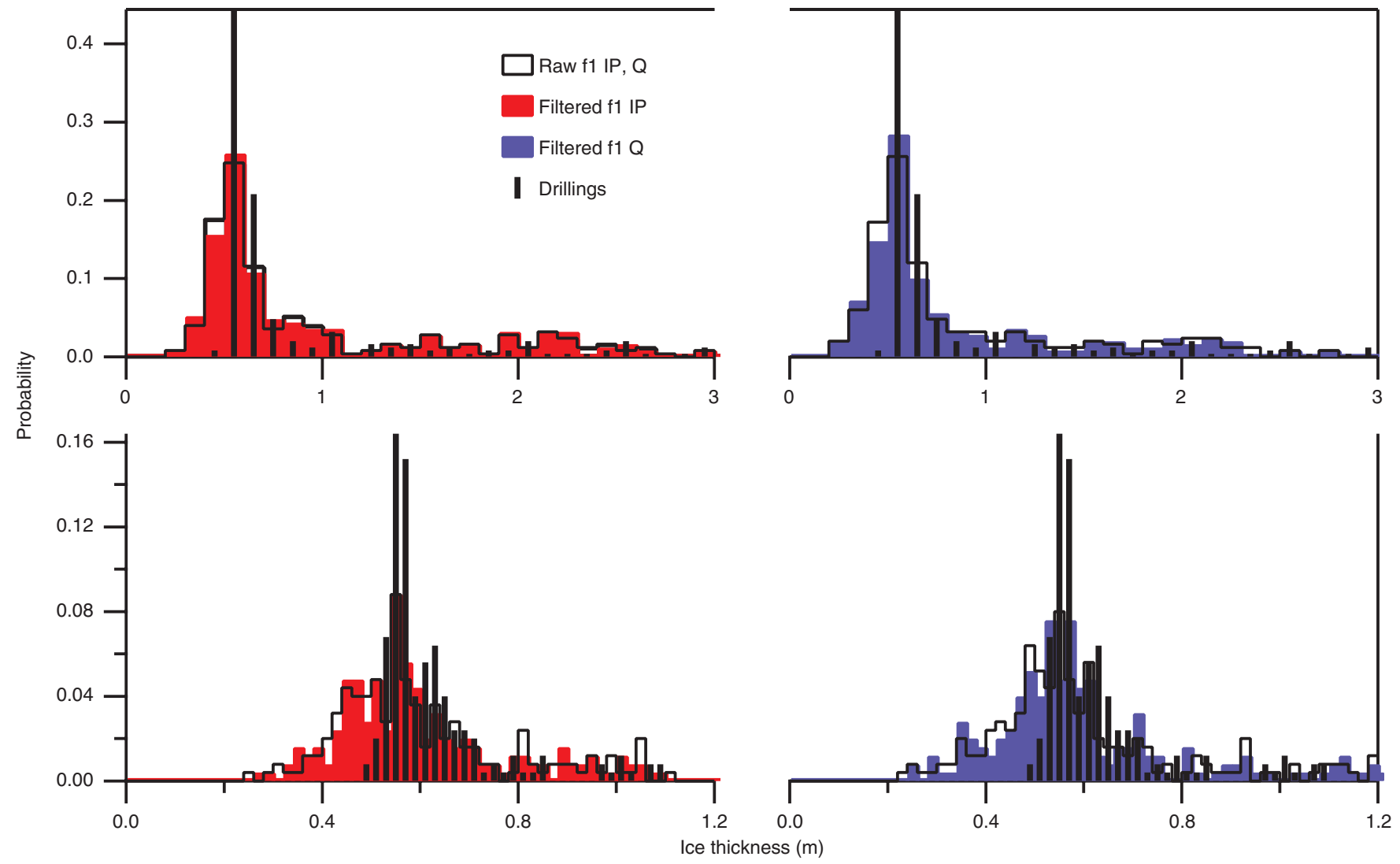

Figure 9. ARISE 2003 field data: ice thickness distributions of drilling data compared to raw and filtered EMPEX thickness estimates for 3.68-kHz IP and Q. Histograms in the upper row use 10-cm bins; the lower row shows a close-up with 2-cm bins. 
3.68-kHz IP and Q. Otherwise, the consistency of distinct thickness estimates may act as an indicator of $3 \mathrm{D}$ features in the vicinity. Note that on the flanks of the main pressure ridge in Figure 8, IP and Q diverge because of the smaller footprint of Q compared to IP.

In contrast to the option of several thickness results for one data point using EMPEX, formal layered-earth inversion would yield one thickness estimate representing all available data. Further, full inversion would appear capable of accounting for anomalous ice conductivity, shallow bathymetry, and bird attitude effects. Operationally, however, EMPEX represents the favorable processing scheme, especially for the described AWI-HEM system for instrumental/technical reasons: The component chosen for EMPEX processing (3.68 kHz IP) is characterized by the highest sensitivity with respect to ice thickness and the lowest instrumental noise level. Incorporating any other component (e.g., using least-squares inversion) would degrade the resulting ice thickness precision and accuracy.

\section{CONCLUSIONS}

A 1D, approximate direct HEM inversion algorithm is described and evaluated. We introduce an analytical definition for sensitivity with respect to sea-ice thickness. This enables us to quantify precision and accuracy estimates for HEM ice thickness mapping. Biases resulting from approximations included in the described EMPEX processing algorithm define the system's accuracy. For generally expected sea-ice conditions, EMPEX accuracy is within the desired $10 \mathrm{~cm}$. Synthetic precision, governed by instrumental noise, is better than $10 \mathrm{~cm}$, given small noise levels $(<5 \mathrm{ppm})$ and sensor altitudes $(\sim 15 \mathrm{~m})$ for small-scale system geometries as used in sea-ice HEM.

Besides these quantitative specifications, EMPEX ice thickness estimates may be degraded by 3D sea-ice geometries, such as pressure ridges, and rare phenomena, such as highly conductive gap layers within the ice floe or shallow water in the area of investigation. Layered-earth inversion potentially could account for the latter.

The derived half-space sensitivities are a valuable measure for comparing the performance of HEM instruments. Utilizing sensitivity values, technical noise specifications in parts per million can be transferred to model-space precision estimates in centimeters. The field data example confirms the synthetically stated precision estimates $(12 \mathrm{~cm})$ when studying the retrieved level-ice thickness preci$\operatorname{sion}(9 \mathrm{~cm})$.

The most dominant cause of occasional poor accuracy for EMPEX sea-ice thickness appears to be the unaccounted for pitch-androll movements of the HEM bird. If attitude measurements were available, attitude effects could be corrected, transforming distorted EM fields to HMD fields and tilted laser altitudes to nadir measures. This way, attitude-corrected data could be fed through the EMPEX transform, still avoiding elaborate layered-earth inversion.

Our results confirm the EMPEX transform as a useful, very stable, and fast tool for ground-, ship- and airborne EM sea-ice thickness profiling.

\section{ACKNOWLEDGMENTS}

Part of this research was funded by the EC research project SITHOS. We thank the ARISE 2003 shipboard party for conducting almost 1000 drill-hole measurements of sea-ice thickness, the officers and crew of the RV Aurora Australis for field support, and the pilots and technicians of Helicopter Resources Pty Ltd. Insightful comments from three anonymous reviewers, the associate editor, and the editor, substantially improved the manuscript.

\section{REFERENCES}

Abramowitz, M., and I. A. Stegun, 1964, Handbook of mathematical functions with formulas, graphs, and mathematical tables: Dover Publications. ACIA, 2004, Impacts of a warming Arctic, Arctic climate impact assessment: Cambridge University Press.

Bergeron, C. J., Jr., 1986, Modified image method: Application to the response of layered ohmic conductors to active electromagnetic sources: Journal of Applied Physics, 59, 3901-3908.

Bergeron, C. J., J. W. Ioup, and G. A. Michel, 1987, Lateral resolution of the modified image method for sea-ice thickness: 57th Annual International Meeting, SEG, Expanded Abstracts, Session EM1.3.

, 1989, Interpretation of airborne electromagnetic data using the modified image method: Geophysics, 54, 1023-1030.

Brent, R. P., 1973, Algorithms for minimization without derivatives: Prentice-Hall.

Bryan, M. W., K. W. Holladay, C. J. Bergeron, J. W. Ioup, and G. E. Ioup, 2003, MIM and nonlinear least-squares inversions of AEM data in Barataria basin, Louisiana: Geophysics, 68, 1126-1131.

Guptasarma, D., and B. Singh, 1997, New digital linear filters for Hankel J0 and J1 transforms: Geophysical Prospecting, 45, 745-762.

Haas, C. 2004, Late-summer sea ice thickness variability in the Arctic transpolar drift 1991-2001 derived from ground-based electromagnetic sounding: Geophysical Research Letters, 31, L09402; .

Haas, C., S. Gerland, H. Eicken, and H. Miller, 1997, Comparison of sea-ice thickness measurements under summer and winter conditions in the Arctic using a small electromagnetic induction device: Geophysics, 62, 749-757.

Holladay, J. S., B. Lo, and S. J. Prinsenberg, 1997, Bird orientation effects in quantitative airborne electromagnetic interpretation of pack ice thickness sounding: Oceans Conference 1997, Marine Technology Society/Institute of Electrical and Electronics Engineers, Proceedings, vol. 2, 1114-1119.

Holladay, J. S., J. R. Rossiter, and A. Kovacs, 1990, Airborne measurements of sea-ice thickness using electromagnetic induction sounding: 9th Offshore Mechanics and Arctic Engineering Conference, Proceedings, 309-315.

Kovacs, A., and J. S. Holladay, 1990, Sea ice thickness measurement using a small airborne electromagnetic sounding system: Geophysics, 55, 1327-1337.

Kovacs, A., J. S. Holladay, and C. J. J. Bergeron, 1995, The footprint/altitude ratio for helicopter electromagnetic sounding of sea ice thickness: Comparison of theoretical and field estimates: Geophysics, 60, 374-380.

Kovacs, A., N. C. Valleau, and J. S. Holladay, 1987, Airborne electromagnetic sounding of sea-ice thickness and subice bathymetry: Cold Regions Science and Technology, 14, 289-311.

Liu, G., and A. Becker, 1990, Two-dimensional mapping of sea ice keels with airborne electromagnetics: Geophysics, 55, 239-248.

Liu, G., A. Kovacs, and A. Becker, 1991, Inversion of airborne electromagnetic survey data for sea-ice keel shape: Geophysics, 56, 1986-1991.

Massom, R., A. P. Worby, V. Lytle, T. Markus, I. Allison, T. Scambos, H. Enomoto, K. Tateyama, T. Haran, J. Comiso, A. Pfaffling, T. Tamura, A Muto, P. Kanagaratnam, B. Giles, N. Young, G. Hyland, and E. Key, 2006 , ARISE (Antarctic Remote Ice Sensing Experiment) in the East: Validation of satellite-derived sea-ice data products: Annals of Glaciology, 44, 288-296.

Meju, M., 1994, Geophysical data analysis: Understanding inverse problem theory and practice: SEG.

Morey, R. M., A. Kovacs, and G. F. N. Cox, 1984, Electromagnetic properties of sea ice: Cold Regions Science and Technology, 9, 53-75.

Multala, J., H. Hautaniemi, M. Oksama, M. Lepparanta, J. Haapala, A. Herlevi, K. Riska, and M. Lensu, 1996, An airborne system on a fixed-wing aircraft for sea ice thickness mapping: Cold Regions Science and Technology, 24, 355-373.

Peterson, I. K., S. J. Prinsenberg, and J. S. Holladay, 1999, Using a helicopter-borne EM-induction system to validate RADARSAT sea ice signatures: 28th International Port and Ocean Engineering under Arctic Conditions (POAC) Conference, Proceedings, vol. 1, 275-284.

Prinsenberg, S. J., J. S. Holladay, and J. Lee, 2002, Measuring ice thickness with EISFlow ${ }^{\mathrm{TM}}$, a fixed-mounted helicopter electromagnetic-laser system: 12th International Offshore and Polar Engineering Conference, Proceedings, vol. 1,737-740.

Reid, J. E., A. Pfaffling, and J. Vrbancich, 2006a, Airborne electromagnetic footprints in 1D earths: Geophysics, 71, no. 2, G63-G72.

Reid, J. E., A. Pfaffling, A. P. Worby, and J. R. Bishop, 2006b, In situ measurements of the direct-current conductivity of Antarctic sea ice: Implications for airborne electromagnetic sounding of sea-ice thickness: Annals of Glaciology, 44, 217-223.

Reid, J. E., J. Vrbancich, and A. P. Worby, 2003, A comparison of shipborne 
and airborne electromagnetic methods for Antarctic sea ice thickness measurements: Exploration Geophysics, 34, 46-50.

Schauer, U., and G. Kattner, 2004, The Expedition ARKTIS XIX/1 a,b and XIX/2 of the Research Vessel "POLARSTERN" in 2003: Reports on Polar and Marine Research, 481, Alfred Wegener Institute for Polar and Marine Research.

Smith, B. D., D. V. Smith, P. L. Hill, and V. F. Labson, 2003, Helicopter electromagnetic and magnetic survey data and maps, Seco Creek area, Medina and Uvalde counties, Texas: U.S. Geological Survey. Open-File Report 03-226.

Stroeve, J., M. C. Serreze, F. Fetterer, T. Arbetter, W. Meier, J. Maslanik, and K. Knowles, 2005, Tracking the Arctic's shrinking ice cover: Another ex- treme September sea ice minimum in 2004: Geophysical Research Letters, 32, L04501

Timco, G. W., 1979, An analysis of the in-situ resistivity of sea ice in terms of its microstructure: Journal of Glaciology, 22, 461-471.

Thyssen, F., H. Kohnen, M. V. Cowan, and G. W. Timco, 1974, DC resistivity measurements on the sea ice near Pond Inlet, N. W. T. (Baffin Island): Polarforschung, 44, 117-126.

Valleau, N. C., 2000, HEM data processing - A practical overview: Exploration Geophysics, 31, 584-594.

Ward, S. H., and G. W. Hohmann, 1988, Electromagnetic theory for geophysical applications, in M. N. Nabighian, ed., Electromagnetic methods in applied geophysics, vol. 1, Theory: SEG, 131-311. 